\title{
Exacerbation frequency and clinical outcomes in adult patients with cystic fibrosis
}

\author{
Kaïssa de Boer, ${ }^{1}$ Katherine L Vandemheen, ${ }^{1}$ Elizabeth Tullis, ${ }^{2}$ Steve Doucette, ${ }^{1}$ \\ Dean Fergusson, ${ }^{1}$ Andreas Freitag, ${ }^{3}$ Nigel Paterson, ${ }^{4}$ Mary Jackson, ${ }^{5}$ \\ M Diane Lougheed, ${ }^{6}$ Vijay Kumar, ${ }^{7}$ Shawn D Aaron ${ }^{1}$
}

\begin{abstract}
${ }^{1}$ The Ottawa Hospital Research Institute, University of Ottawa, Ottawa, Ontario, Canada ${ }^{2}$ University of Toronto, Toronto, Ontario, Canada

${ }^{3}$ McMaster University, Hamilton, Ontario, Canada

${ }^{4}$ University of Western Ontario, London, Ontario, Canada ${ }^{5}$ Grand River Hospital, Kitchener, Ontario, Canada ${ }^{6}$ Queen's University, Kingston, Ontario, Canada

${ }^{7}$ Sudbury Regional Hospital, Sudbury, Ontario, Canada
\end{abstract}

\section{Correspondence to} Shawn D Aaron, The Ottawa Hospital, General Campus, University of Ottawa, 501 Smyth Road, Ottawa, Ontario, Canada K1H 8L6;

saaron@ohri.ca

Received 7 February 2011 Accepted 26 May 2011 Published Online First 15 June 2011

\section{ABSTRACT \\ Background Despite advances in treatment of cystic fibrosis (CF), pulmonary exacerbations remain common. The aim of this study was to determine if frequent pulmonary exacerbations are associated with greater declines in lung function, or an accelerated time to death or lung transplantation in adults with CF. \\ Methods A 3-year prospective cohort study was conducted on 446 adult patients with CF from Ontario, Canada who could spontaneously produce sputum. Patients enrolled from 2005 to 2008 and were stratified into groups based upon their exacerbation rates over the 3 year study: $<1$ exacerbation/year $(n=140), 1-2$ exacerbations/year $(n=160)$ and $>2$ exacerbations/year $(n=146)$. Exacerbations were defined as acute/subacute worsening of respiratory symptoms severe enough to warrant oral or intravenous antibiotics. Patient-related factors associated with frequent exacerbations were determined, and clinical outcomes were compared among the three exacerbation groups.}

Results Patients with frequent exacerbations were more likely to be female, diabetic and have poorer baseline lung function. Patients with $>2$ exacerbations/year had an increased risk of experiencing a $5 \%$ decline from baseline forced expiratory volume in $1 \mathrm{~s}\left(\mathrm{FEV}_{1}\right)$; unadjusted HR 1.47 (95\% Cl 1.07 to 2.01, $p=0.02$ ), adjusted HR 1.55 (95\% Cl 1.10 to 2.18, $p=0.01)$ compared with patients with $<1$ exacerbation/year. Patients with $>2$ exacerbations/year also had an increased risk of lung transplant or death over the 3 year study; unadjusted HR 12.74 (95\% Cl 3.92 to 41.36 , $\mathrm{p}<0.0001)$, adjusted HR 4.05 (95\% Cl 1.15 to 14.28 , $\mathrm{p}=0.03$ ).

Conclusions Patients with CF with frequent exacerbations appear to experience an accelerated decline in lung function, and they have an increased 3 year risk of death or lung transplant.

\section{INTRODUCTION}

Despite advances in understanding the molecular basis and the development of new treatments for cystic fibrosis (CF), pulmonary exacerbations continue to contribute to significant clinical burden among patients with CF. In a large observational study of $>11000$ patients, $42 \%$ of patients with CF experienced a pulmonary exacerbation over a 6 month period. ${ }^{1}$ Although currently there are no consensus diagnostic criteria which define a pulmonary exacerbation, typically a pulmonary exacerbation is diagnosed based upon a constellation of patient symptoms and signs including dyspnoea,

\section{Key messages}

What is the key question?

- Do adults with cystic fibrosis (CF) who experience frequent pulmonary exacerbations have greater declines in lung function or an accelerated progression to death or lung transplant?

\section{What is the bottom line?}

- Patients with CF with frequent pulmonary exacerbations appear to experience an accelerated decline in lung function, and they have an increased 3 year risk of death or lung transplant.

\section{Why read on?}

- To learn about the potential impact of CF pulmonary exacerbations on the overall health status of adults with CF.

cough, sputum production, decreased energy level and appetite, weight loss and decreases in lung function. $^{2}$ Treatment usually involves commencement of intravenous or oral antibiotics, and intensification of sputum clearance via chest physiotherapy. ${ }^{3}$

Several studies have shown that pulmonary exacerbations negatively impact on quality of life in patients with $\mathrm{CF}^{4-6}$ and are associated with significant cost. ${ }^{7}$ Pulmonary exacerbations of CF, chronic obstructive pulmonary disease (COPD) and asthma are clearly serious events and have recently been coined 'lung attacks'. ${ }^{8}$ Studies have shown that the annual prevalence of CF pulmonary exacerbations increases with age. ${ }^{19}$ Despite advances in CF care, pulmonary exacerbation rates have not declined over the last two decades. ${ }^{10}$

Studies using data from the US CF Patient registry have found a negative correlation between pulmonary exacerbation rates and values of forced expiratory volume in $1 \mathrm{~s}\left(\mathrm{FEV}_{1}\right)$, suggesting that lung function is lower in patients who experience more exacerbations. ${ }^{2}$ A small retrospective singlecentre study of 51 patients recently suggested that $\mathrm{FEV}_{1}$ decline is associated with the number of pulmonary exacerbations/year. ${ }^{11}$

It has been hypothesised that exacerbations might induce greater airway inflammation and provoke subsequent accelerated declines in lung function ${ }^{11}{ }^{12}$; however, this theory has not been conclusively proven. Our hypothesis was that 
patients with frequent pulmonary exacerbations might experience greater long-term morbidity. The objective of our study was to determine if frequent pulmonary exacerbations are associated with greater declines in lung function and body mass index (BMI), and an accelerated time to death or lung transplantation in adults with CF.

\section{METHODS \\ Study design}

This was a secondary analysis of a 3 year prospective observational cohort study involving an inception cohort of adult patients with CF from Ontario, Canada who could spontaneously produce sputum at the time of entry. The original study was designed to determine whether infection with transmissible strains of Pseudomonas aeruginosa was associated with poor clinical outcomes. Primary clinical outcomes in the original study included pulmonary exacerbation rates, survival and lung function, and these same outcomes are presented here in the current study. ${ }^{13}$

\section{Patients and data collection}

Adult patients attending each of the seven Ontario adult CF clinics were approached for participation in the study between September 2005 and September 2008. Patients were included if they were $\geq 18$ years of age and had a confirmed diagnosis of CF with genetic testing and/or sweat testing. Patients were excluded if they were unable to produce sputum at enrolment (since the primary objective of the original study was to examine transmissible strains of $P$ aeruginosa). The research ethics boards of the participating centres approved the study, and participants provided written informed consent.

Pulmonary exacerbations were defined as acute or subacute worsening of respiratory symptoms severe enough to warrant oral or intravenous treatment with antibiotics. Treatment was at the discretion of the treating physicians.

Patients were seen at baseline and annually for three consecutive years. At each visit we collected height, weight, BMI, spirometry and exacerbation history. Information on exacerbations and exacerbation treatment was collected each year for events in the preceding 12 month period. Exacerbation events were ascertained through a review of the patient's clinic and hospital charts. Direct patient interviews were conducted at baseline and then annually to determine if there were exacerbations that had not been captured in the health record. We specifically collected data on: number of pulmonary exacerbations in the past year requiring oral or intravenous antibiotics, number of inpatient pulmonary exacerbations requiring intravenous antibiotics, number of pulmonary exacerbations treated exclusively at home with intravenous antibiotics and number of pulmonary exacerbations treated exclusively on an outpatient basis with oral antibiotics.

We also collected data on baseline chronic infections at the time of study entry, including infection with both unique and transmissible strains of $P$ aeruginosa. $P$ aeruginosa transmissible strains are genetically identical strains that infect unrelated patients with CF. Co-morbidities (pancreatic insufficiency, CFrelated diabetes and CF-related liver disease) and medical treatments were recorded from each patient at baseline and at each subsequent follow-up clinic visit.

Patients who underwent lung transplantation before completion of the study did not contribute spirometry, BMI or exacerbation data after their lung transplant date. Spirometry was performed according to American Thoracic Society (ATS) standards, and predicted values from Crapo et al were used. ${ }^{14}$ Patients completed measurements of lung function at each annual prescheduled visit (0, 12, 24 and 36 months).

\section{Statistical analysis}

Patients were grouped according to their mean exacerbation status over the 3 year follow-up period: (1) $<1$ exacerbation/ year; (2) 1-2 exacerbations/year; and (3) $>2$ exacerbations/year. The groupings were made a priori before commencing study analysis, based on the distribution of the data so that three approximately equal sized groups could be assigned. We selected three exacerbation groupings to determine if there was a graded response to pulmonary exacerbations. Once established, the exacerbation groupings were used as predefined cut-off points and subsequent analyses were run using only these cut-off points. Baseline continuous variables between the groups were compared using analysis of variance (ANOVA) and categorical variables were compared using $\chi^{2}$ tests as appropriate, with $p$ values reflecting the difference between the three groups (table 1).

We assessed decline in lung function using two methods. The slope of the rate of decline of $\mathrm{FEV}_{1} \%$ predicted over the 3 year study period was compared between the reference group $(<1$ exacerbation/year) and the other two groups using random effects mixed linear models. Group-time interactions were analysed using the SAS PROC.MIXED program. Potential confounding effects of age, sex, BMI, infection with Burkholderia cepacia complex and transmissible $P$ aeruginosa strains, baseline $\mathrm{FEV}_{1} \%$ predicted, CF co-morbidities (diabetes, liver disease and pancreatic insufficiency) and medical treatments (inhaled tobramycin, inhaled colistin, azithromycin, dornase $\alpha$ ) were assessed using linear mixed models. We adjusted all of our analyses for the same covariates which were determined a priori. These covariates were selected because they have previously been shown to be statically associated with either mortality or health outcomes in CF.

We also assessed $\mathrm{FEV}_{1}$ decline by using Cox proportional hazards models to compare the time to $5 \%$ decline of patient's $\mathrm{FEV}_{1} \%$ predicted relative to their baseline lung function in the reference group compared with the other two groups. The time to lung transplant or death was compared using Kaplan-Meier survival methods and Cox proportional hazards models. Mortality was also examined as a stand-alone variable. Lastly, we assessed for trends by determining if the interaction trend between time and group was linear across the three groupings.

We performed a secondary analysis examining the effect of exacerbations requiring intravenous antibiotics on rate of decline of $\mathrm{FEV}_{1}$ and time to lung transplant or death. Patients were again stratified based on mean annual number of exacerbations requiring intravenous antibiotics over the 3 year study: (1) 0 exacerbation/year; (2) 0.01-0.99 exacerbations/year; and 3) $\geq 1$ exacerbations/year. Statistical analyses were performed using the same methods as described above. All statistical testing was two-sided and was performed at the 5\% significance level using SAS software version 9.0

\section{RESULTS}

\section{Patient selection and baseline characteristics}

Five hundred and eighty patients were approached to enter the study, and in total 446 patients enrolled (figure 1). Of the 446 enrolled patients, 140 patients averaged $<1$ exacerbation/year, 160 patients had 1-2 exacerbations/year and 146 patients had $>2$ exacerbations/year during the study period. These patients 
Table 1 Patient baseline characteristics

\begin{tabular}{|c|c|c|c|c|}
\hline & $\begin{array}{l}<1 \text { Exacerbation/ } \\
\text { year } n=140\end{array}$ & $\begin{array}{l}1-2 \text { Exacerbations/ } \\
\text { year } n=160\end{array}$ & $\begin{array}{l}>2 \text { Exacerbations/ } \\
\text { year } n=146\end{array}$ & $\begin{array}{l}\text { Between-group } \\
\text { p value }\end{array}$ \\
\hline Average follow-up time, years & 2.17 & 2.27 & 2.34 & \\
\hline Total no. of exacerbations & 158 & 710 & 1512 & \\
\hline Mean age (SD), years & $28.5 \pm 9.7$ & $29.1 \pm 9.1$ & $30.2 \pm 10.1$ & 0.33 \\
\hline Sex $(\%$ male $)$ & $96(68.6 \%)$ & $92(57.5 \%)$ & $67(45.9 \%)$ & 0.0005 \\
\hline Mean height (SD), cm & $169.8 \pm 9.4$ & $169 \pm 9.3$ & $167.6 \pm 8.3$ & 0.11 \\
\hline \multicolumn{5}{|l|}{ Lung function } \\
\hline $\mathrm{FEV}_{1}$ measured $(\mathrm{SD})$, litres & $2.70 \pm 1.00$ & $2.26 \pm 0.87$ & $1.81 \pm 0.85$ & $<0.0001$ \\
\hline $\mathrm{FEV}_{1} \%$ predicted (SD) & $67.5 \pm 20.7$ & $59.0 \pm 20.8$ & $49.1 \pm 20.4$ & $<0.0001$ \\
\hline FVC measured (SD), litres & $4.71 \pm 0.86$ & $5.47 \pm 0.83$ & $4.37 \pm 0.76$ & 0.002 \\
\hline FVC \% predicted (SD) & $83.4 \pm 17.8$ & $77.77 \pm 20.6$ & $67.9 \pm 21.6$ & $<0.0001$ \\
\hline Staphylococcus aureus & $39(28.7 \%)$ & $44(27.7 \%)$ & $37(25.5 \%)$ & 0.83 \\
\hline Stenotrophomonas maltophilia & $6(4.4 \%)$ & $14(8.8 \%)$ & $15(10.3 \%)$ & 0.17 \\
\hline Transmissible Pseudomonas aeruginosa strains & $42(30.0 \%)$ & $28(17.5 \%)$ & $32(21.9 \%)$ & 0.11 \\
\hline Pseudomonas aeruginosa (all strains) & $101(72.1 \%)$ & $101(68.8 \%)$ & $109(74.7 \%)$ & 0.67 \\
\hline \multicolumn{5}{|l|}{ Co-morbidities, n (\%) } \\
\hline CF-related diabetes & $23(16.4 \%)$ & $28(17.5 \%)$ & $41(28.1 \%)$ & 0.02 \\
\hline Pancreatic insufficiency & $116(82.9 \%)$ & $132(82.5 \%)$ & $127(87 \%)$ & 0.50 \\
\hline CF-related liver disease & $4(2.9 \%)$ & $8(5 \%)$ & $11(7.5 \%)$ & 0.20 \\
\hline \multicolumn{5}{|l|}{ Medications, n (\%) } \\
\hline Azithromycin & $30(21.4 \%)$ & $52(32.5 \%)$ & $61(41.8 \%)$ & 0.001 \\
\hline Inhaled colistin & $5(3.6 \%)$ & $8(5 \%)$ & $16(11 \%)$ & 0.03 \\
\hline Dornase $\alpha$ & $10(7.1 \%)$ & $16(10 \%)$ & $31(21.2 \%)$ & 0.0007 \\
\hline
\end{tabular}

$\mathrm{BMI}$, body mass index; $\mathrm{CF}$, cystic fibrosis; $\mathrm{FEV}_{1}$, forced expiratory volume in $1 \mathrm{~s}$; $\mathrm{FVC}$, forced vital capacity.

were followed for a mean of 791, 827 and 855 days, respectively. Full 3 year follow-up was not available for 101 patients 50 with $<1$ exacerbation/year, 32 with 1-2 exacerbations/year and 19 with $>2$ exacerbations/year), who enrolled in the study after January 2007. Late enrollees were mostly paediatric patients who transitioned to adult CF clinics during the latter half of the study. These patients were followed until December 2009. Vital status (death or lung transplant) was assessed for all enrolled

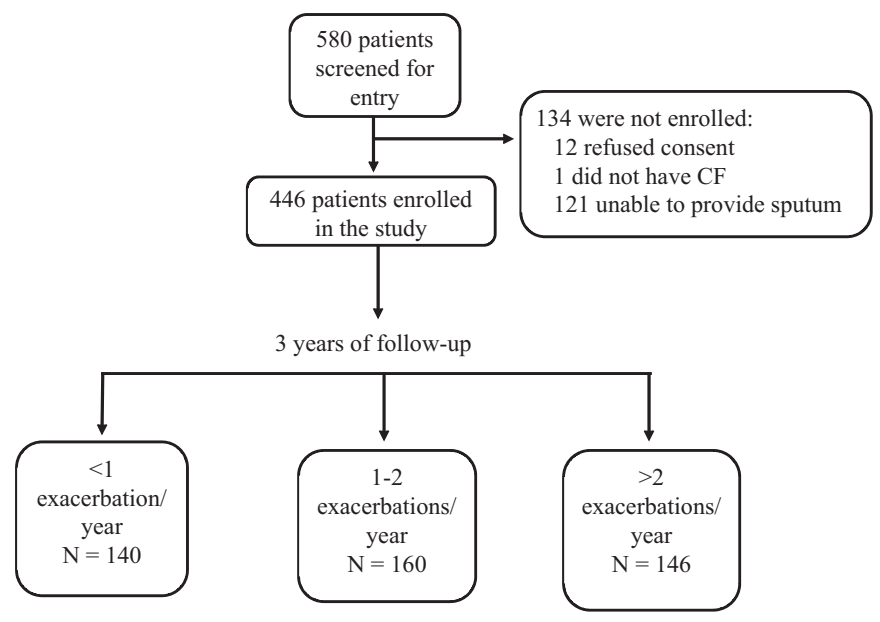

Figure 1 Flow diagram of entry of patients into the study. CF, cystic fibrosis. patients 3 years after entry into the study or, for those who enrolled late, up to 31 December 2009.

Table 1 outlines the baseline characteristics of patients included among the three pulmonary exacerbation groupings. Patients with frequent exacerbations were more likely to be female, diabetic and have poorer baseline lung function.

\section{Exacerbation frequency and decline in $\mathrm{FEV}_{\mathbf{1}}$ and $\mathrm{BMI}$}

Patients with more frequent exacerbations had lower lung function at entry into the study, and all three groups experienced a decline in their $\mathrm{FEV}_{1}$ over the course of the study. Over the 3 year study the mean decline from baseline in $\mathrm{FEV}_{1} \%$ predicted was $-4.85 \%$ (95\% CI $-8.01 \%$ to $-1.69 \%)$ for patients with $<1$ exacerbation/year, $-5.44 \% \quad(95 \%$ CI $-8.33 \%$ to $-2.55 \%$ ) for patients with $1-2$ exacerbations/year, and $-6.49 \%$ (95\% CI $-10.10 \%$ to $-2.91 \%$ ) for patients with $>2$ exacerbations/year. The slopes of rate of decline were not significantly different between the three groups and the $p$ value for trend across the three groups was not significant $(p=0.36)$.

The analysis of rate of decline in $\mathrm{FEV}_{1}$ was also undertaken using survival analysis to try to correct for differences in between-group rates of premature censoring of patients who experienced death or lung transplant. Sixty-seven (48\%) patients with $<1$ exacerbation/year, 93 (58\%) patients with 1-2 exacerbations/year and 93 (64\%) patients with $>2$ exacerbations/ year experienced a $\geq 5 \%$ decline in $\mathrm{FEV}_{1}$ from baseline. Compared with patients who had $<1$ exacerbation/year, patients with 1-2 exacerbations/year did not have a significantly increased risk of experiencing a $5 \%$ decline in $\mathrm{FEV}_{1} \%$ 
predicted from baseline over the study period; unadjusted HR 1.29 (95\% CI 0.95 to $1.77, \mathrm{p}=0.11$ ), adjusted HR 1.33 (95\% CI 0.96 to $1.84, \mathrm{p}=0.08)$. However, patients with $>2$ exacerbations/ year did experience a more rapid progression to $5 \%$ decline in $\mathrm{FEV}_{1} \%$ predicted from baseline compared with patients with $<1$ exacerbation/year; unadjusted HR 1.47 (95\% CI 1.07 to 2.01, $\mathrm{p}=0.02$ ), adjusted HR 1.55 (95\% CI 1.10 to $2.18, \mathrm{p}=0.01$ ) (table 2). The $\mathrm{p}$ value for trend was statistically significant across the three groups $(p=0.02)$.

There was no difference in change in BMI when comparing patients with $<1$ exacerbation/year versus $1-2$ exacerbations/ year (unadjusted difference in decline of BMI of 0.14 per year, $95 \%$ CI -0.1 to 0.46 ) or for patients with $<1$ exacerbation/year versus $>2$ exacerbations/year (unadjusted difference in decline of BMI of 0.04 per year, $95 \%$ CI -0.29 to 0.35 ). The $p$ value for trend for BMI was non-significant $(p=0.89)$.

Exacerbation frequency and risk of death or lung transplantation Over the 3 year study period 50/446 (11.2\%) patients died or received a lung transplant. All deaths were respiratory deaths. Death or lung transplant occurred in $3 / 140$ (2.1\%) patients in the $<1$ exacerbation/year group, $11 / 160$ (6.9\%) in the $1-2$ exacerbations/year group and $36 / 146(24.7 \%)$ in the $>2$ exacerbations/year group. Patients with $>2$ exacerbations/year experienced a greater 3 year risk of death or lung transplant compared with patients with $<1$ exacerbation/year; unadjusted HR 12.74 (95\% CI 3.92 to 41.36, p<0.0001), adjusted HR 4.05 (95\% CI 1.15 to $14.28, p=0.03$ ). Patients with $1-2$ exacerbations/year did not experience a significantly accelerated risk of death or lung transplant versus those with $<1$ exacerbation/ year; unadjusted HR 3.25 (95\% CI 0.91 to 11.66, $\mathrm{p}=0.07$ ), adjusted HR 2.02 (95\% CI 0.53 to $7.73, \mathrm{p}=0.30$ ) (figure 2).

\section{Exacerbation frequency and risk of death}

A Kaplan-Meier analysis of time to death was performed, independently of lung transplant. Death occurred during the 3 year study follow-up period in $2 / 140$ (1.4\%) patients in the $<1$ exacerbation/year group, $4 / 160(2.5 \%)$ in the $1-2$ exacerbations/ year group and $16 / 146(11.0 \%)$ in the $>2$ exacerbations/year group (Kaplan-Meier log rank $p$ value $=0.0002$ ). Patients with $>2$ exacerbations/year did experience an increased 3 year risk of death compared with patients with $<1$ exacerbation/year; unadjusted HR 7.86 (95\% CI 1.81 to 34.2, $\mathrm{p}=0.006$ ). However, patients with 1-2 exacerbations/year did not have a statistically increased risk of death compared with patients with $<1$ exacerbation/year; unadjusted HR 1.73 (95\% CI 0.32 to 9.45 , $\mathrm{p}=0.53)$.

\section{Secondary analysis of exacerbations requiring intravenous antibiotics}

$\mathrm{FEV}_{1} \%$ predicted was significantly lower at baseline in patients who experienced more frequent exacerbations requiring intra-

Table 2 Analysis of time to $5 \%$ decline of baseline $\mathrm{FEV}_{1} \%$ predicted

\begin{tabular}{lll}
\hline & Unadjusted HR & Adjusted $\mathbf{H R}^{*}$ \\
\hline$<1$ exacerbation/year & 1.29 & 1.33 \\
versus & $95 \% \mathrm{Cl} \mathrm{0.95}$ to 1.77 & $95 \% \mathrm{Cl} 0.96$ to 1.84 \\
$1-2$ exacerbations/year & $\mathrm{p}=0.11$ & $\mathrm{p}=0.08$ \\
$<1$ exacerbation/year & 1.47 & 1.55 \\
versus & $95 \% \mathrm{Cl} 1.07$ to 2.01 & $95 \% \mathrm{Cl} 1.10$ to 2.18 \\
$>2$ exacerbations/year & $\mathrm{p}=0.02$ & $\mathrm{p}=0.01$ \\
\hline
\end{tabular}

*Adjusted for age, sex, body mass index, infection with Burkholderia cepacia, transmissible Pseudomonas aeruginosa, baseline forced expiratory volume in $1 \mathrm{~s}\left(\mathrm{FEV}_{1}\right)$, cystic fibrosis co-morbidities and maintenance therapies.

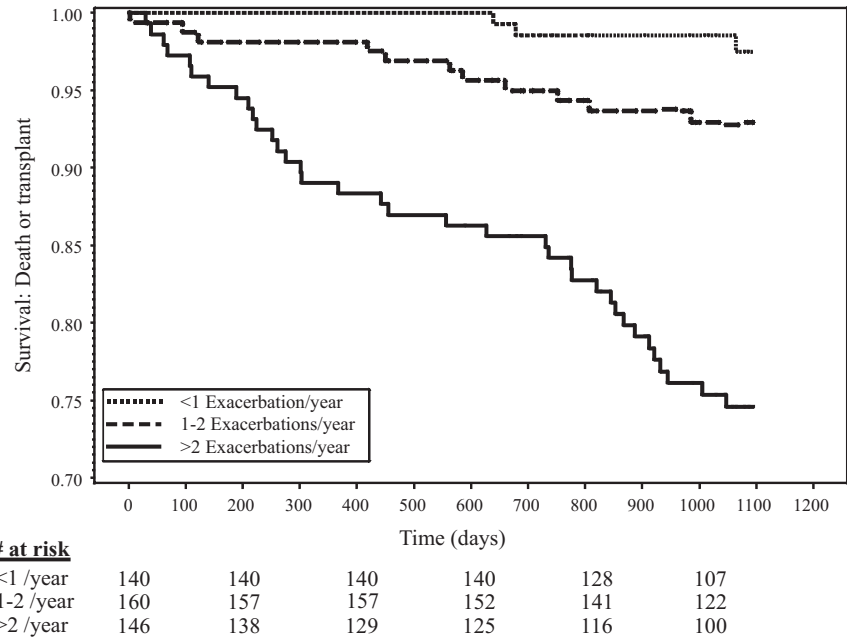

Figure 2 Kaplan-Meier plot comparing time to death or lung transplant over the 3 year study period for exacerbation groups. Survival analyses were adjusted for age, sex, body mass index, infection with Burkholderia cepacia, transmissible Pseudomonas aeruginosa, baseline forced expiratory volume in $1 \mathrm{~s}\left(\mathrm{FEV}_{1}\right)$, cystic fibrosis co-morbidities and maintenance therapies.

venous antibiotics. The baseline $\mathrm{FEV}_{1} \%$ predicted was $67.4 \%$ in the 0 intravenous exacerbation/year group, $56.6 \%$ in the 0.01-0.99 intravenous exacerbations/year group and $42.0 \%$ in those with $\geq 1$ intravenous exacerbations/year; $p<0.0001$.

Compared with patients who had 0 intravenous exacerbations/year, patients with CF with $0.01-0.99$ intravenous exacerbations/year had a significantly increased risk of experiencing a $5 \%$ decline in $\mathrm{FEV}_{1} \%$ predicted from baseline over the study period; unadjusted HR 1.45 (95\% CI 1.08 to $1.95, \mathrm{p}=0.01$ ), adjusted HR 1.58 (95\% CI 1.15 to $2.16, \mathrm{p}=0.004)$. Patients with $\geq 1$ intravenous exacerbations/year also experienced a more rapid progression to $5 \%$ decline in $\mathrm{FEV}_{1} \%$ predicted from baseline compared with patients with 0 intravenous exacerbation/year; unadjusted HR 1.46 (95\% CI 1.08 to $1.97, \mathrm{p}=0.02$ ), adjusted HR 1.78 (95\% CI 1.24 to $2.54, \mathrm{p}=0.002$ ).

Death or lung transplant occurred in $6 / 228$ patients $(2.6 \%)$ in the 0 intravenous exacerbation/year group, $5 / 104$ patients $(6.9 \%)$ in the 0.01-0.99 intravenous exacerbations/year group and 39/ $114(34.2 \%)$ patients in the $\geq 1$ intravenous exacerbations/year group. Patients requiring $\geq 1$ intravenous course of antibiotics/ year experienced a significantly increased 3 year risk of death or transplant compared with patients with 0 intravenous exacerbation/year; unadjusted HR 15.68 (95\% CI 6.64 to 37.06, $\mathrm{p}<0.0001)$, adjusted HR 3.36 (95\% CI 1.30 to $8.73, p=0.01$ ). Patients requiring 0.01-0.99 intravenous exacerbations/year did not experience an increased 3 year risk of death or lung transplant compared with patients with 0 intravenous exacerbations/ year; unadjusted HR 1.77 (95\% CI 0.54 to $5.80, p=0.35$ ), adjusted HR 0.93 (95\% CI 0.27 to $3.15, p=0.90$ ) (figure 3).

\section{DISCUSSION}

The results of our study suggest that patients with CF who experience frequent pulmonary exacerbations are at increased risk of experiencing a decline in their lung function by $5 \%$ from baseline and that frequent pulmonary exacerbations are associated with an increased 3 year risk of death or lung transplant. Patients who experienced $>2$ exacerbations/year were also at significantly increased risk for death, independent of lung transplant. The results were similar whether we considered 


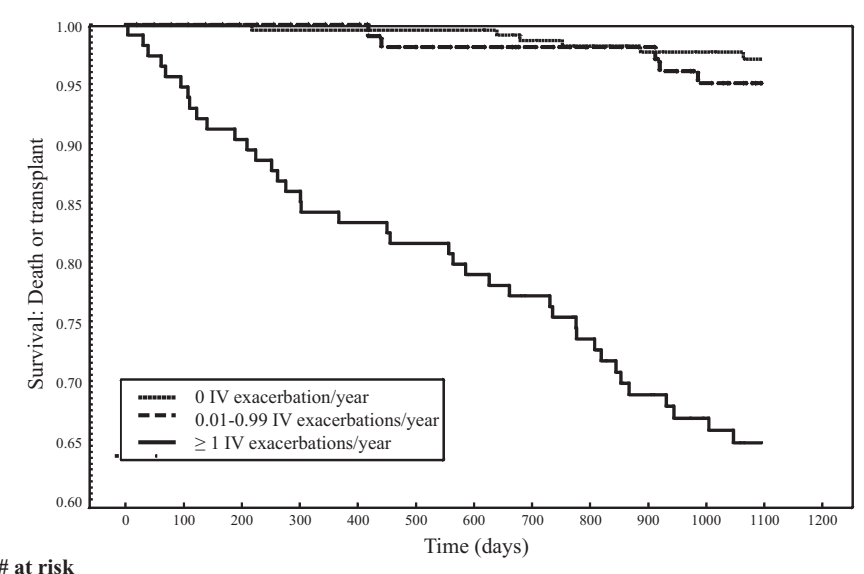

\# at risk

$\begin{array}{lll}0 / \text { year } & 228 & 228 \\ 0.01-0.99 / \text { year } & 104 & 104\end{array}$

$0.01-0.99$ year $104 \quad 104$

$\geq 1$ /year $\quad 114$

104
103

227
104
95

226
102

207

174

95

$\begin{array}{ll}98 & 90 \\ 80 & 65\end{array}$
Figure 3 Kaplan-Meier plot comparing time to death or lung transplant over the 3 year study period for patients with pulmonary exacerbations requiring intravenous (IV) antibiotics. Survival analyses were adjusted for age, sex, body mass index, infection with Burkholderia cepacia, transmissible Pseudomonas aeruginosa, baseline forced expiratory volume in $1 \mathrm{~s}\left(\mathrm{FEV}_{1}\right)$, cystic fibrosis co-morbidities and maintenance therapies.

exacerbations that were treated with either oral or intravenous antibiotics, or when we restricted our analysis to exacerbations treated exclusively with intravenous antibiotics. To our knowledge, ours is the first study to quantify the rate of $\mathrm{FEV}_{1}$ decline and the RR of mortality and lung transplant in relation to exacerbation frequency.

Analysis of the annual rate of decline in $\mathrm{FEV}_{1}$ in this study was made difficult by 'floor effects' and by 'healthy survivor effects'. Patients who experienced a mean of $>2$ exacerbations/ year over the course of the 3 year study were clearly a sicker cohort of patients with lower $\mathrm{FEV}_{1}$ upon entry into the study (mean $\mathrm{FEV}_{1} 49 \%$ predicted). Konstan et al found that paediatric patients with higher $\mathrm{FEV}_{1}$ experienced the steepest declines in lung function. ${ }^{9}$ Taking this finding into consideration, it is possible that lower baseline $\mathrm{FEV}_{1}$ values may introduce a floor effect whereby patients' $\mathrm{FEV}_{1}$ cannot decline at a steep rate since they are already at the lower end of the distribution curve. Potentially even more important for our analysis was 'the healthy survivor effect'. Patients who died or had lung transplants did not contribute annual lung function data following these events. Since a much greater proportion of patients who experienced $>2$ exacerbations/year died or had lung transplants, censoring of these sickest patients from this cohort resulted in apparently lower rates of decline in lung function among the healthy survivors.

Our analysis of lung function decline was therefore undertaken in two ways. We analysed the rate of annual decline in $\mathrm{FEV}_{1} \%$ predicted but we also undertook a survival analysis to try to correct for differences in between-group rates of premature censoring of patients. Using a survival analysis of time to a $5 \%$ decline in $\mathrm{FEV}_{1} \%$ predicted from baseline allowed us to incorporate premature censoring of patient data due to death or lung transplant, and this analysis did show that patients with $>2$ exacerbations/year did experience a more rapid progression in $\mathrm{FEV}_{1}$ decline from baseline compared with infrequent exacerbators.

We found that patients with frequent pulmonary exacerbations were more likely to be female and diabetic. Female gender has been previously identified as a risk factor for pulmonary exacerbations ${ }^{15}$ and poorer survival outcomes, ${ }^{16}$ as has diabetes. ${ }^{17-19}$

We assessed each patient's average exacerbation rate over 3 years as the independent variable in this analysis. This allowed for a more robust, longer term estimate of patients' propensity for exacerbations. However, a potential limitation of our study was our method of defining exacerbations. Pulmonary exacerbations remain a subjective diagnosis, and a unifying definition of pulmonary exacerbations is still lacking within the CF literature. ${ }^{2} 20$ We used an event-based definition, and we defined exacerbations as acute/subacute worsening of patients' respiratory symptoms severe enough to warrant oral or intravenous treatment with antibiotics. Our definition can be considered a 'real world' definition, and we did not require a prescribed minimum number of signs or symptoms for an event to be considered an exacerbation.

Our study excluded non-sputum-producing patients, and the study results are therefore generalisable only to the $75-80 \%$ of adult patients with CF who spontaneously produce sputum. Lastly, our study showed an association between exacerbation frequency and time to lung transplant or death; however, our study was not designed to determine causality. For instance, frequent pulmonary exacerbations may predispose a patient to lung transplant or, alternatively, patients listed for lung transplant may be more aggressively treated and therefore appear to have more exacerbations.

We demonstrated that more frequent pulmonary exacerbations in patients with CF are associated with a significantly higher risk of death or lung transplant. Patients with $>2$ pulmonary exacerbations/year have reduced lung function at baseline which continues to decline progressively over time. Patients with CF who regularly experience $>2$ pulmonary exacerbations/year are clearly at high risk, and they warrant diligent clinical monitoring and timely consideration for lung transplant.

Acknowledgements We thank Lesley Gaskin, and Jennifer Pike (St. Michael's Hospital, Toronto, Ontario), Ena Gaudet, RN and Kathleen Devecseri, RN (The Ottawa Hospital, Ottawa, Ontario), Rosamund Hennessey, RN (McMaster University, Hamilton, Ontario), Tracey Gooyers, RN, Patrice Kean, BScN and Jennifer Itterman, BScN (University of Western Ontario, London, Ontario), Sharri-Lynne Zinger, RN and Charlene Piche, RN (Sudbury Regional Hospital, Sudbury, Ontario), Lori Peterson, RN, BScN, MS (Grand River Hospital, Kitchener, Ontario) and Lisa Smith RN, BScN, MSc (Queen's University, Kingston, Ontario) for study coordination, and My-Linh Tran and Jennie Cote (The Ottawa Hospital Research Institute) for assistance with data management.

Funding The Canadian Institutes of Health Research, Canadian Cystic Fibrosis Foundation and Ontario Thoracic Society. The funding sources had no role in the design and conduct of the study, analysis or interpretation of the data, preparation or final approval of the manuscript, or the decision to submit the manuscript for publication.

\section{Competing interests None.}

Ethics approval This study was conducted with the approval of the The Ottawa Hospital, and the other six hospitals that were involved in this study.

Contributors All of the listed authors contributed to the design and analysis and write-up of the manuscript.

Provenance and peer review Not commissioned; externally peer reviewed.

\section{REFERENCES}

1. Rabin HR, Butler SM, Wohl ME, et al; Epidemiologic Study of Cystic Fibrosis. Pulmonary exacerbations in cystic fibrosis. Pediatr Pulmonol 2004;37:400-6.

2. Goss $\mathbf{C H}$, Burns JL. Exacerbations in cystic fibrosis. 1: epidemiology and pathogenesis. Thorax 2007:62:360-7.

3. Regelmann WE, Elliott GR, Warwick WJ, et al. Reduction of sputum Pseudomonas aeruginosa density by antibiotics improves lung function in cystic fibrosis more than do bronchodilators and chest physiotherapy alone. Am Rev Respir Dis 1990;141:914-21. 
4. Britto MT, Kotagal UR, Hornung RW, et al. Impact of recent pulmonary exacerbations on quality of life in patients with cystic fibrosis. Chest 2002;121:64-72.

5. Dobbin CJ, Bartlett D, Melehan K, et al. The effect of infective exacerbations on sleep and neurobehavioral function in cystic fibrosis. Am J Respir Crit Care Med 2005;172:99-104.

6. Goldbeck L, Zerrer S, Schmitz TG. Monitoring quality of life in outpatients with cystic fibrosis: feasibility and longitudinal results. J Cyst Fibros 2007;6:171-8.

7. Robson M, Abbott J, Webb $\mathrm{K}$, et al. A cost description of an adult cystic fibrosis unit and cost analyses of different categories of patients. Thorax 1992;47:684-9.

8. FitzGerald JM. Targeting lung attacks. Thorax 2011;66:365-6.

9. Konstan MW, Morgan WJ, Butler SM, et al; Scientific Advisory Group and the Investigators and Coordinators of the Epidemiologic Study of Cystic Fibrosis. Risk factors for rate of decline in forced expiratory volume in one second in children and adolescents with cystic fibrosis. J Pediatr 2007;151:134-9.

10. Cystic Fibrosis Foundation Patient Registry 2008. Annual Report to the Center Directors. Bethesda, MD: Cystic Fibrosis Foundation, 2009.

11. Amadori A, Antonelli A, Balteri I, et al. Recurrent exacerbations affect FEV(1) decline in adult patients with cystic fibrosis. Respir Med 2009;103:407-13.

12. Jarad NA, Higgs $S$, Jeffcote $T$, et al. Factors associated with reduced FEV1 in adult patients with cystic fibrosis in a relatively affluent area. Chron Respir Dis 2005:2:133-7.
13. Aaron SD, Vandemheen $\mathrm{KL}$, Lewis-Giesbrecht $\mathrm{T}$, et al. Infection with transmissible strains of Pseudomonas aeruginosa and clinical outcomes in adults with cystic fibrosis. JAMA 2010;19:2145-53.

14. Crapo RO, Morris AH, Gardner RM. Reference spirometric values using techniques and equipment that meet ATS recommendations. Am Rev Respir Dis 1981;123:659-64.

15. Block JK, Vandemheen KL, Tullis $\mathrm{E}$, et al. Predictors of pulmonary exacerbations in patients with cystic fibrosis infected with multi-resistant bacteria. Thorax 2006:61:969-74

16. Liou TG, Adler FR, Fitzsimmons SC, et al. Predictive 5-year survivorship model of cystic fibrosis. Am J Epidemiol 2001;153:345-52.

17. Marshall BC, Butler SM, Stoddard M, et al. Epidemiology of cystic fibrosis-related diabetes. J Pediatr 2005;146:681-7.

18. Jarad NA, Giles K. Risk factors for increased need for intravenous antibiotics for pulmonary exacerbations in adult patients with cystic fibrosis. Chron Respir Dis 2008;5:29-33.

19. Amin R, Dupuis A, Aaron SD, et al. The effect of chronic infection with Aspergillus fumigatus on lung function and hospitalization in patients with cystic fibrosis. Chest 2010;137:171-6.

20. Flume PA, Mogayzel PJ Jr, Robinson KA, et al; Clinical Practice Guidelines for Pulmonary Therapies Committee. Cystic fibrosis pulmonary guidelines: treatment of pulmonary exacerbations. Am J Respir Crit Care Med 2009:180:802-8.

\section{Journal club}

\section{Oxygen therapy is equivalent to room air for alleviating refractory dyspnoea}

Palliative oxygen is commonly used for the treatment of dyspnoea in individuals with lifelimiting illness who are ineligible for long-term oxygen therapy despite insufficient evidence for symptomatic benefit. This multicentre study investigated the effectiveness of oxygen compared with room air in this population group.

Between 2006 and 2008, 239 patients from Australia, the USA and the UK were randomly assigned to receive at least $15 \mathrm{~h}$ of oxygen $(n=120)$ or room air $(n=119)$ for 7 days. The primary outcome measured was 'breathlessness' using a 0-10 numerical rating scale recorded by the patient twice daily.

There was no significant difference in breathlessness between the two groups. Symptomatic improvement was appreciable in the first $72 \mathrm{~h}$ of intervention. A greater response was seen in the morning with oxygen therapy than with room air, but the response remained the same in the evening for both groups. The side effects of treatment and change in quality of life were similar in the two groups.

The authors conclude that oxygen is not superior to room air in palliative treatment of breathlessness; however, this study is limited by the lack of representation of the sickest patients in palliative care as only outpatients were recruited. In ineffective cases, alternative measures should be sought as medical gas therapy is restricted by cost, availability and logistic burdens.

Abernethy AP, McDonald CF, Frith PA, et al. Effects of palliative oxygen versus room air in relief of breathlessness in patients with refractory dyspnoea: a double-blinded, randomised controlled trial. Lancet 2010;376:784-93.

\section{Joyce R Y Chew}

Correspondence to Joyce R Y Chew, Foundation Year 2, General Medicine, Ayr Hospital, Dalmellington Road, Ayr, KA6 6DX, UK; jcruoyi@gmail.com

Published Online First 12 November 2011

Thorax 2011;66:685. doi:10.1136/thx.2010.153502 